\title{
The Effect of a Western Diet on Hepatic Autophagy in Age Accelerated SAMP8 Mice
}

\author{
MATTHEW P. HARRIS1,2, MEGAN S. SCHALLER 1,2, DAVID UMBAUGH ${ }^{1,2}$, KARIN \\ SANDOVAL 2 , KEN WITT2 2 , and JOSHUA S. WOOTEN ${ }^{1,2}$
}

1. Exercise Physiology and Biochemistry Lab; Department of Applied Health; Southern Illinois University Edwardsville; Edwardsville, IL. 2. Department of Pharmaceutical Sciences; Southern Illinois University Edwardsville; Edwardsville, IL

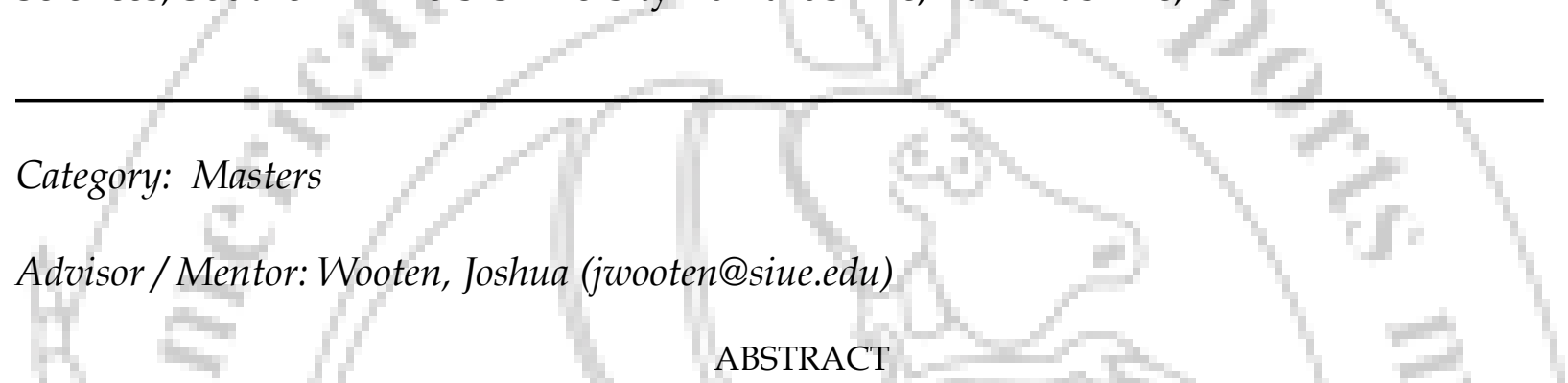

Non-alcoholic steatohepatitis (NASH) is characterized as a dysregulation of hepatic lipid metabolism and a chronic inflammatory state. It is hypothesized the link between lipid dysregulation and inflammation may be due in part to defective hepatic autophagy and reduced mitochondrial capacity to oxidize fatty acids. It remains to be determined; however, the effects of a Western diet on hepatic autophagy and mitochondrial function during aging. PURPOSE: The purpose of this study was to determine the effect of a high-fat high fructose diet (HFF) on markers of hepatic autophagy and mitochondrial function in an age accelerated mouse model. METHODS: Twenty week old, male and female, SAMP8 mice $(n=49)$ were randomly assigned, matching for gender, to either a standard chow (SC) or HFF (45\% fat, $24 \%$ fructose) diet for 32 weeks. Liver tissue was analyzed for mRNA expression of autophagic (BNIP3, Beclin 1, p62, and Atg7) and mitochondrial (PGC1a and COXIV) genes. Differences between gender and dietary groups were identified by a $2 \times 2$ ANOVA and statistical significance was set at $\mathrm{p}<0.05$. RESULTS: Following 32 weeks of feeding, male mice fed the HFF diet were significantly heavier than male mice in the SC group ( $31.6 \mathrm{~g}$ vs $26.5 \mathrm{~g}$; $\mathrm{p}=0.001$ ); however, no difference was observed between diet groups for female mice. The HFF diet resulted in higher autophagic activity as observed by Beclin $1(+36 \% ; p=0.001)$ and $\mathrm{BNIP3}(+40 \%$; $\mathrm{P}=0.003)$ expression. Despite the higher autophagic activity, p62 was higher $(+31 \% ; \mathrm{p}<0.001)$ in the HFF compared to the SC group, suggesting impaired autophagic flux. In addition, mitochondrial COXIV expression was elevated $(+43 \%$; $\mathrm{P}<0.001)$ in the HFF group compared to the SC group suggesting increased $\beta$-oxidation. Overall, the expression of all autophagic and mitochondrial markers was higher in male compared to female mice; however, both sexes responded similarly to the HFF diet. CONCLUSION: Despite the higher expression of autophagic and mitochondrial genes, elevated expression of p62 suggests an impaired autophagic flux in age accelerated mice following a Western diet.

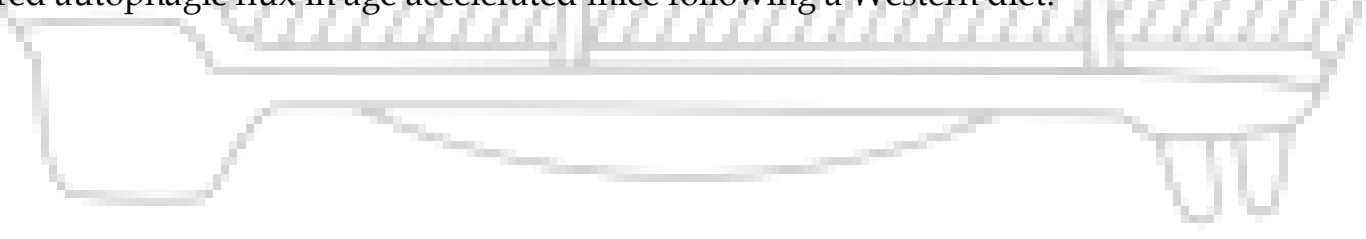

